\title{
Der Arzt und Philosoph Tiberios Klaudios Menekrates
}

Von Jost Benedum

Dem an der ärztlichen Prosopographie der Antike interessierten Leser hat A. A. Tsaknakis ${ }^{1}$ im ersten Heft der Historia Medicinae Veterinariae den Änderungsvorschlag von $\mathrm{L}$. Moretti ${ }^{2}$ bezüglich einer nunmehr seit fast siebzig Jahren bekannten Arztinschrift zugänglich gemacht. Die Grabinschrift, die von einer Marmorplatte aus Mermere ${ }^{3}$ in Lydien stammt und von J. Keil und A. von Premerstein ${ }^{4} 1908$ erstmals veröffentlicht wurde, bietet folgenden Text:

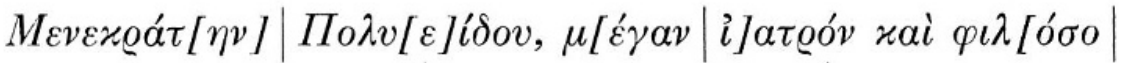

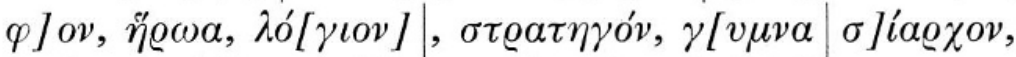

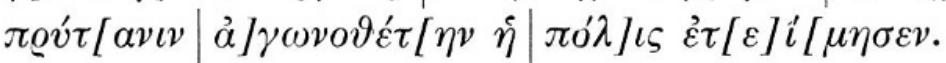

Die Übersetzung lautet:

Die Stadt ehrte Menekrates, den Sohn des Polyeides, den großen Arzt und Philosophen, den Heros, Logisten, Strategen, Gymnasiarchen, Agonotheten ${ }^{5}$.

Bislang herrschte Einvernehmen darüber, daß, von gewissen Änderungsmöglichkeiten abgesehen ${ }^{6}$, die vorliegende Inschrift einen im 1. Jahrhundert $n$. Chr. bekannten Arzt erwähnt, der nach seinem Tode als Heros geehrt wurde. Fraglich war lediglich, ob dieser Arzt Menekrates mit einem der bisher aus literarischen und epigraphischen Zeugnissen bekannten Ärzte gleichen Namens identifiziert werden kann. Solche Zuordnungs- oder Trennungsversuche stoßen zwar nicht selten auf große Schwierigkeiten, doch rechtfertigt dies keinen agnostizistischen Standpunkt. Erst jüngst konnte C. Fabricius ${ }^{7}$ zeigen, daß selbst bei den zahlreichen Ärzten mit Namen «Apollonios» eine weitergehende Unterscheidung möglich ist. Im folgenden sei daher der Versuch unternommen, den Arzt und Philosophen Menekrates anhand der erhaltenen Zeugnisse näher zu betrachten.

Ausgangspunkt ist die oben zitierte Arbeit von L. Moretti, der Anstoß an der

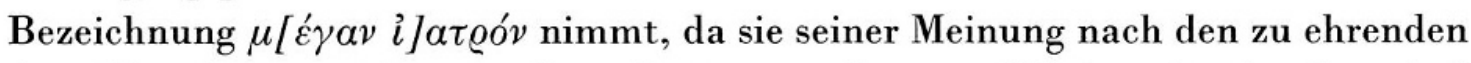
Arzt Menekrates nicht mit der gehörigen und sonst üblichen Ausdrucksweise ${ }^{8}$

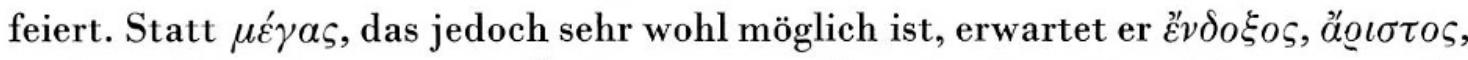
$\tau \varrho \tilde{\omega} \tau o \varsigma$. Daher bietet er als Änderungsvorschlag eine Berufsbezeichnung an, die bis jetzt durch kein Zeugnis belegt ist: $\mu[o v \lambda o \iota] \alpha \tau \varrho o ́ \varsigma$. Demnach wäre der Geehrte ein Tierarzt und das Wort der erste Beleg aus dem Griechischen für

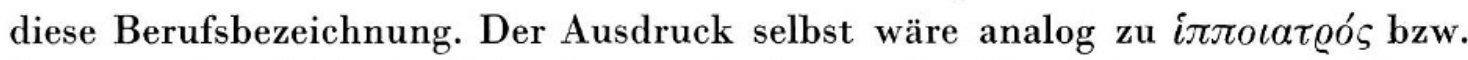
$i \pi \pi \omega \iota \alpha \tau \varrho o ́ \varsigma$ gebildet ${ }^{9}$ und direkt von der lateinischen Bezeichnung mulomedicus übernommen. 
Für solche Übernahme des lat. mulus, mula (Maulesel) und mulio (Mauleseltreiber usf.) ins Griechische ist seit längerem eine Reihe von Belegen ${ }^{10}$ aus Papyri und Inschriften bekannt. Da sich der Änderungsvorschlag auf sie stützt, müssen sie hier kurz angeführt werden.

Wie bereits Fr. Preisigke ${ }^{11}$ notierte, begegnet der Ausdruck $\mu o v \lambda i \omega v$ in einem Papyrus des 4. Jahrhunderts n. Chr., ebenso $\mu о v^{\prime} \lambda \eta$ im Papyrus Leipzig ${ }^{12}$ Nr. 97, der eine Rechnung aus Hermonthis betrifft und nach der Konsulardatierung in das Jahr 338 n. Chr. gehört. Dem Anfang des 4. Jahrhunderts entstammen zwei inschriftliche Belege, die im Edikt Diokletians ${ }^{13}$ über Maximaltarife aus dem Jahr $301 \mathrm{n}$. Chr. stehen. Es handelt sich um die Bezeichnungen $\varkappa \alpha \lambda i \varkappa \omega \nu \mu o v \lambda \iota \omega v \iota \varkappa \tilde{o} v$ (caligae mulionicae: Maultiertreiberstiefel) und [ $\mu o v] \lambda \iota^{\prime} \omega \nu \iota$ (mulioni) ${ }^{14}$. Die wichtigste Stelle ${ }^{15}$, die L. Moretti nicht heranzieht, lautet :

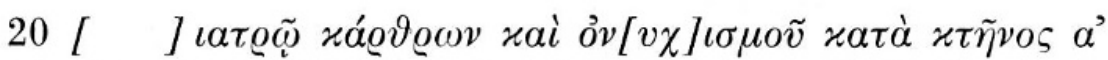

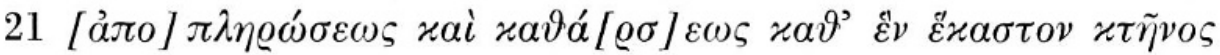

20 Mulomedico tonsurae et aptaturae pedum in capite uno

21 Depleturae et purgaturae capitis per singula capita ${ }^{16}$.

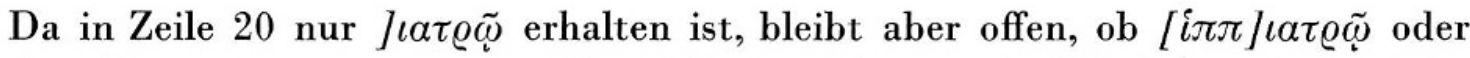
$[\mu o v \lambda] \iota \alpha \tau \varrho \tilde{\omega}$ zu ergänzen ist. Alle bisherigen Herausgeber ${ }^{17}$ haben sich dabei für die gewöhnliche griechische Bezeichnung [i $\pi \pi] \iota \alpha \tau \varrho \tilde{\omega}$ entschieden, obwohl bereits 1888 J. Schmidt ${ }^{18}[\mu o v \lambda] \iota \alpha \tau \varrho \tilde{\omega}$ für «sehr wohl möglich» gehalten hatte, dann aber doch $[\varkappa \tau \eta \nu] \iota \alpha \tau \varrho \tilde{\omega}$ zuneigte, das sich bis heute im Neugriechischen erhalten

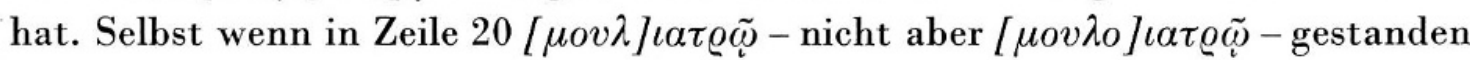
haben sollte, so entstammt dieser Beleg samt den Papyrusurkunden doch erst dem 4. Jahrhundert n. Chr.

Zu den weiteren Belegen, auf die sich der Änderungsvorschlag stützt, zählt ein Steinzeugnis aus Diokaisarea in Kilikien ${ }^{19}$, das in christliche Zeit gehört und zweifelhaft ist. Denn das auf dem Sarkophagdeckel zu lesende $\dagger \mu o v \lambda \alpha \gamma o ́ g o v \dagger$ konnte bislang noch keiner überzeugenden Deutung ${ }^{20}$ zugeführt werden. Selbst wenn auch dieses Zeugnis die Übernahme des lateinischen mul- noch in später Zeit be-

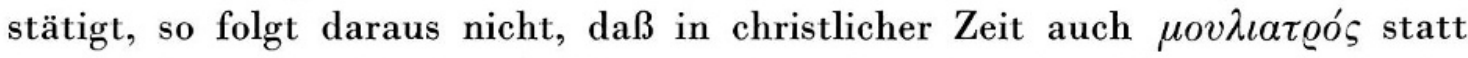

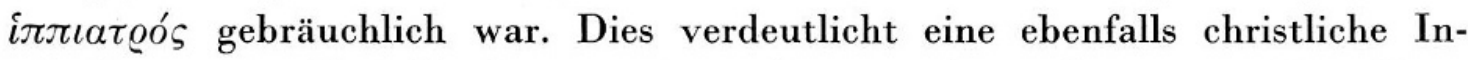

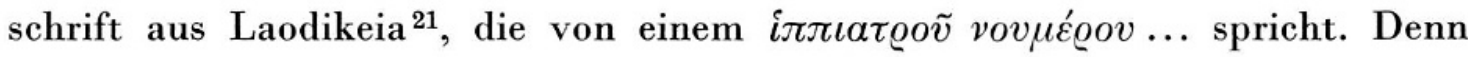
hier hätte man wohl erwarten können, daß neben das direkt aus dem Lateinischen

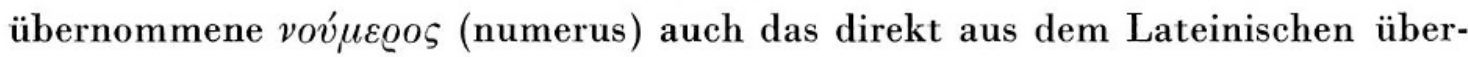
tragene $\mu o v \lambda \iota \alpha \tau \varrho o ́ \varsigma$ (mulomedicus) gesetzt worden wäre.

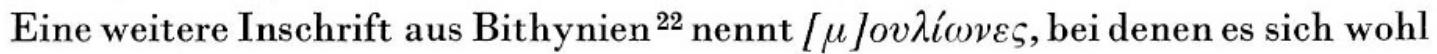
um Maultierkutscher im Postdienst handelt. Nach J. Keil «ist eine Entstehung 


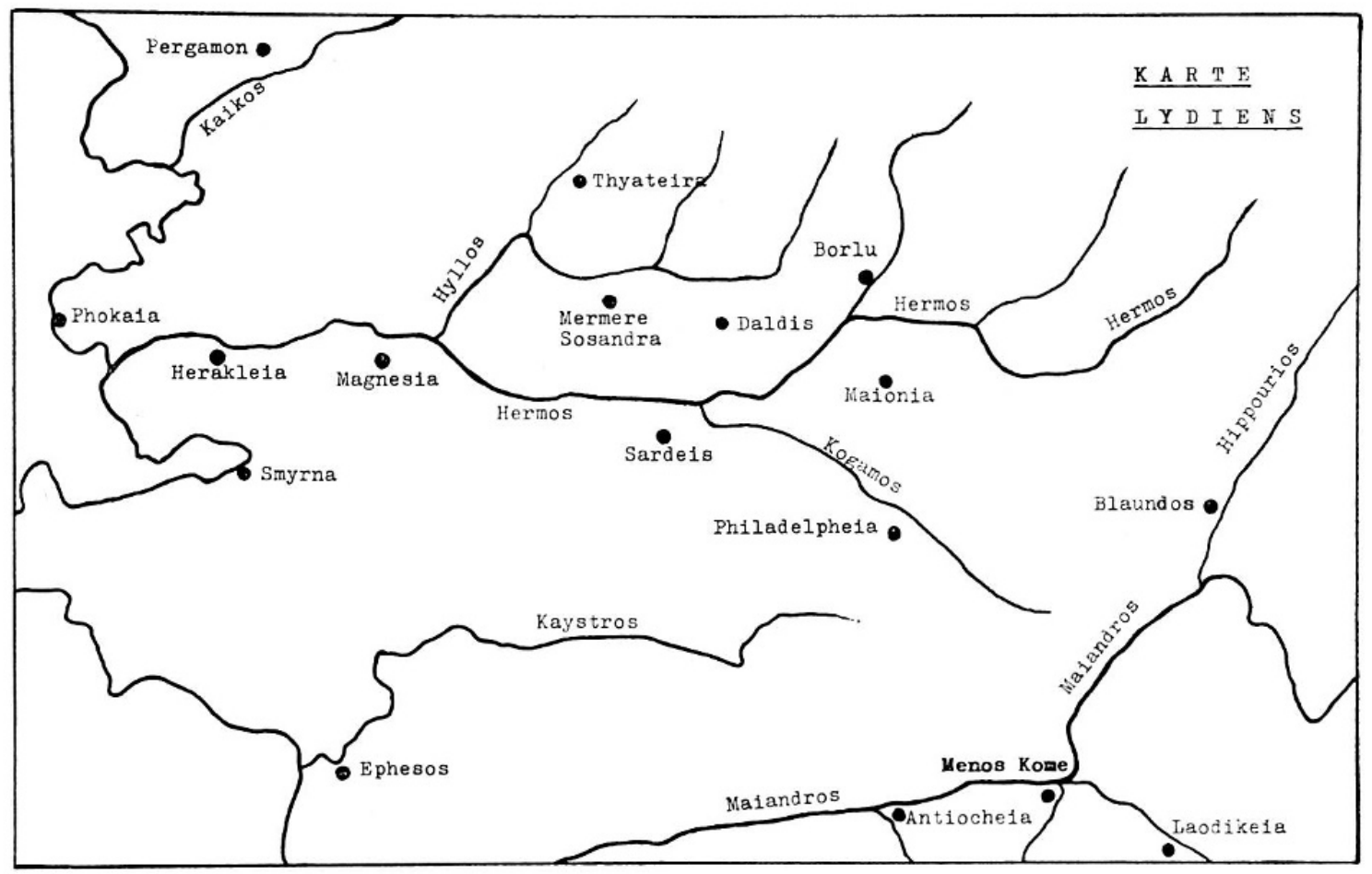

(der Inschrift) vor dem ausgehenden zweiten Jahrhundert n. Chr. durchaus unwahrscheinlich, während ein Herabgehen in das vierte Jahrhundert nicht ausgeschlossen ist ».

Die übrigen Zeugnisse betreffen Berufsbezeichnungen, die z.T. in Personennamen eingegangen sind. So nennt eine Grabinschrift aus Ochrid in Makedonien ${ }^{23}$ einen $\Sigma_{\varepsilon} \beta \eta \varrho \tilde{\nu}$ v dem 3. Jahrhundert n. Chr. angehören. Ferner hat K. Buresch ${ }^{24}$ im Jahre 1898

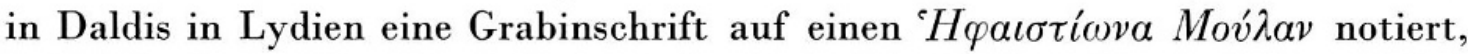
wobei der Name seiner Ansicht nach als « einer der seltsamen Zunamen, an welchen Lydien nicht arm ist », zu werten ist. J. Robert ${ }^{25}$ hat jedoch wahrscheinlich ge-

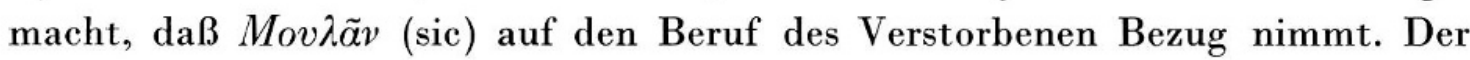
ebenfalls von K. Buresch ${ }^{26}$ auf einem Stein aus Borlu in Lydien gefundene Name

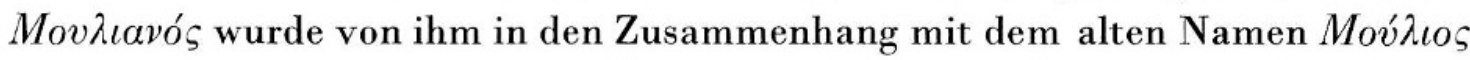
gerückt, den Troer bereits in der Ilias tragen. Wiederum hat dagegen J. Robert ${ }^{27}$

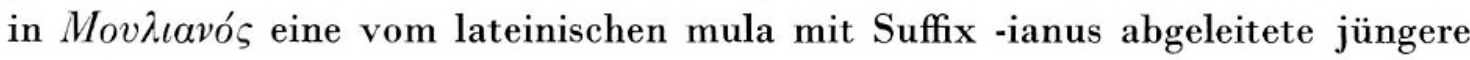
Wortbildung erkannt. Beide lydischen Inschriften gehören nach der Datierung von K. Buresch in das 2. Jahrhundert n. Chr. und stellen somit die frühesten Belege für die Verwendung des lateinischen mul- im Griechischen dar.

Soweit die Zeugnisse. Sie bestätigen, daß das lateinische mulus, mula und mulio direkt in griechische Bezeichnungen übertragen wurden. In keinem Fall ist dabei 
aber die Berufsbezeichnung $\mu о v \lambda \iota \alpha \tau \varrho o ́ \varsigma$ belegt, und das einzige Testimonium des Jahres 301 n. Chr., das hierfür in Anspruch genommen werden könnte, reicht nicht an die Zeit der Menekrates-Inschrift heran. Dies gilt auch für die Steinurkunden aus Lydien, wo das lateinische mul- bereits im 2. Jahrhundert n. Chr. als Berufsmerkmal in griechische Personennamen Eingang gefunden hat. Aus der Tatsache, daß die eingangs zitierte Menekrates-Inschrift ebenfalls aus Lydien stammt, kann aber nicht die innere Begründung dafür abgeleitet werden, daß auch in dieser Inschrift [ $\mu о v \lambda o] \iota \alpha \tau \varrho o ́ \varsigma$ einzusetzen ist. Man muß entgegenhalten, daß

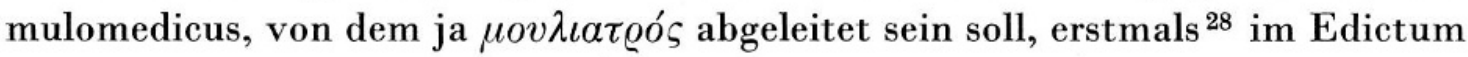
Diocletiani de pretiis vom Jahre $301 \mathrm{n}$. Chr. begegnet, die Inschrift des Arztes Menekrates aber der 1. Hälfte des 1. Jahrhunderts n. Chr. angehört. Damit stimmt überein, daß im eigentlichen veterinärmedizinischen Schrifttum mulomedicus zuerst bei Pelagonius ${ }^{29}$ im 4. Jahrhundert n. Chr. entgegentritt. Der frühe Aus-

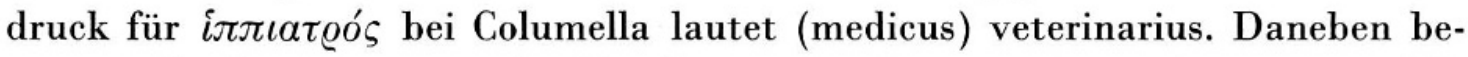
gegnen die Bezeichnungen (medicus) equarius, iumentarius und pecuarius. Die postulierte Übernahme von $\mu o v \lambda \iota \alpha \tau \varrho o ́ s ~ a u s ~ m u l o m e d i c u s ~ k a n n$ nach Ausweis der Belege erst in späterer Zeit stattgefunden haben. Mit gutem Grund wird man da-

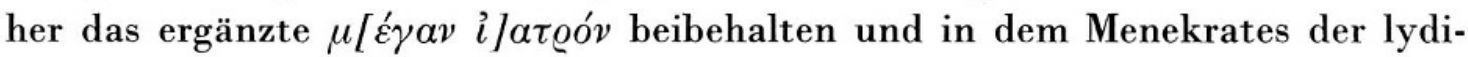
schen Inschrift wie bisher einen Arzt und keinen Tierarzt erblicken.

In dieser Ansicht bestärkt ein weiteres Zeugnis des 1. Jahrhunderts n. Chr., das von der Via Appia bei Rom stammt und ebenfalls einen Arzt Menekrates nennt. Da die Inschrift über die bloße Namens- und Zeitgleichheit ${ }^{30}$ hinaus die Frage nach der Identität der beiden geehrten Ärzte aufwirft, sei sie hier angeführt:

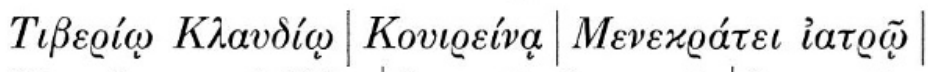

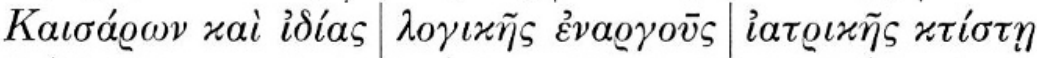

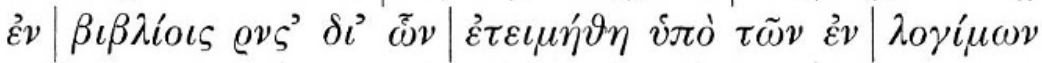

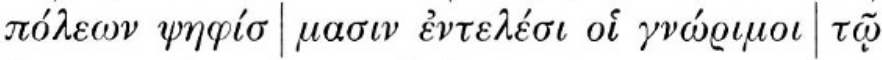

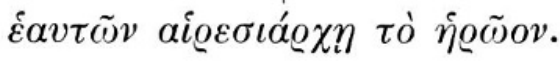

Die Übersetzung lautet:

Dem Tiberios Klaudios Menekrates aus der Tribus Quirina, dem Arzt der Kaiser und dem Begründer eines eigenen logisch-empirisch medizinischen Systems in 156 Büchern, um derentwillen er von namhaften Städten durch Ehrenbeschlüsse geehrt wurde, (setzten) seine Schüler als ihrem Schulhaupt das Heroon.

Bereits J. Keil und A. von Premerstein ${ }^{31}$, die Herausgeber der lydischen Menekrates-Inschrift, haben eine Zuordnung der beiden Ärzte erwogen, sie schließlich aber für wenig wahrscheinlich erklärt ${ }^{32}$. Ihre damalige Begründung, auf die sich noch L. Moretti ${ }^{33}$ beruft, lautet: 
«Es sprechen dagegen vor allem das Fehlen des Titels eines kaiserlichen Leibarztes und die Erwägung, daß die Bekleidung einer ganzen Reihe städtischer Funktionen in der an Stelle Mermeres gelegenen antiken Stadt seitens des berühmten Menekrates, der nach Caelius Aurelianus aus Zeophleta stammte und als Leibarzt in Rom tätig war, sehr geringe Wahrscheinlichkeit für sich hat.»

Zunächst ist festzuhalten, daß die Gleichsetzung mit dem Arzt Menekrates aus Zeophleta ${ }^{34}$, dessen seltsamer Heimatort überdies einer Textverderbnis bei Caelius Aurelianus verdankt wird, irrig ist. Bei diesem Arzt handelt es sich nämlich um Menekrates von Syrakus mit dem anspruchsvollen Beinamen Zeus. Er gehört in die 2. Hälfte des 4. Jahrhunderts v. Chr. ${ }^{35}$ Des weiteren haben kaiserliche Leibärzte mehrfach öffentliche Ämter in Militär, Verwaltung und Kult gerade außerhalb Roms z. B. in ihren Heimat- und Studienorten wahrgenommen, ohne daß ihre Tätigkeit in Rom sie daran gehindert hätte. Dies dürfte auch im vorliegenden Fall gelten, zumal davon auszugehen ist, daß die genannten Ämter weder gleichzeitig noch zeitlebens ausgeübt wurden. Gerade lydische Inschriften zeigen, daß Prytanen und Strategen nicht selten nur wenige Monate im Amt standen. Ganz abgesehen davon, konnte aber ein Amt wie das der Agonothesie auch von einem Ver-

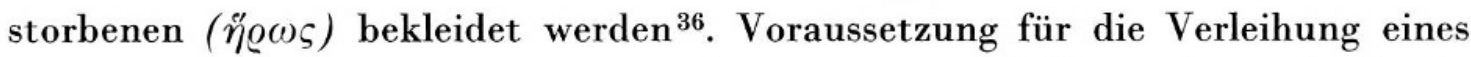
solchen Ehrenamtes war lediglich die Zuwendung von Geldmitteln an die Stadtkasse, die der Verstorbene zu Lebzeiten, testamentarisch oder über seine Hinterbliebenen verfügen konnte. Was schließlich den vermißten Titel eines kaiserlichen Leibarztes in der lydischen Inschrift angeht, so braucht nur auf Markos Artorios Asklepiades, den "Arzt des Kaisers Augustus », hingewiesen zu werden, der auf zwei Inschriften aus Athen und Delos ebenfalls ohne Titel entgegentritt ${ }^{37}$. Die oben zitierte Begründung des Jahres 1908 dürfte somit heute nicht mehr gegen eine Identifizierung der beiden Ärzte geltend gemacht werden können. Angesichts der auffälligen Namens- und Zeitgleichheit stellt sich vielmehr die Frage nach weiteren Anhaltspunkten, die eine Zuordnung wahrscheinlich machen können.

Ein Vergleich der Inschriften ergibt zunächst die Übereinstimmung, daß der rühmliche Titel eines Heros ( $๊ \varrho \omega \alpha$ bzw. $\tilde{\eta} \varrho \tilde{\omega} o v)^{38}$ beiden Ärzten nach ihrem Tode zuteil wurde. Im ersten Fall von der lydischen Stadt ( $\left.\pi o^{\prime} \lambda \iota \varsigma\right)$, im zweiten Fall von den Schülern (oi $\gamma v \omega ́ \varrho \iota \mu о \iota)$ in Rom. Beides schließt eine Identität nicht von vornherein aus. Die Veranlassung für diese Ehrung wird man ferner beidemal in außergewöhnlichen fachlichen Leistungen zu erblicken haben, wofür wiederum der Arzt Markos Artorios Asklepiades sprechen kann, der um 30 n. Chr. von Rat

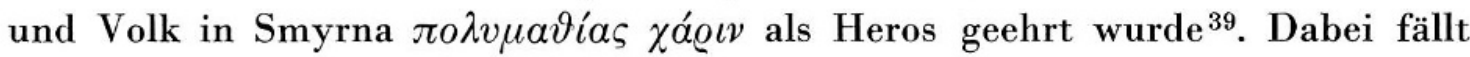

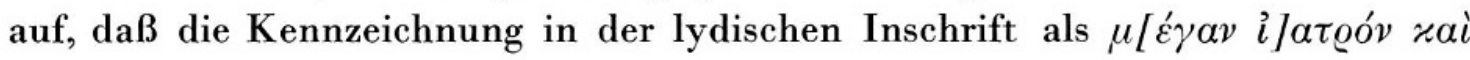


$\varphi \iota \lambda[o ́ \sigma o \varphi] \circ v$ ihre Entsprechung in der Inschrift aus Rom findet. Denn hier ist von einem Ktistes und Hairesiarchen die Rede, der als Verfasser medizinischer Werke vielfach geehrt wurde. Zwar sind außer der lydischen Inschrift, die wohl auf die Heimatstadt zurückgeht, die erwähnten weiteren «Ehrenbeschlüsse namhafter Städte» bislang unbekannt; bekannt ist aber das Lob eines Mannes, der für sich selbst in Anspruch nahm, als Arzt und Philosoph ein medizinisches System geschaffen zu haben. Es ist der sonst gerade Kollegen gegenüber an Lob karge Galen, der sich anerkennend über den Arzt Menekrates äußert ${ }^{40}$. Dabei unterliegt keinem Zweifel, daß der von Galen mehrfach genannte Menekrates der Arzt Tiberios Klaudios Menekrates der Inschrift aus Rom ist ${ }^{41}$, der als $\iota \alpha \tau \varrho o ̀ \varsigma$ $K \alpha \iota \sigma a ́ \varrho \omega \nu$ wie so viele seiner Kollegen in dieser Zeit eine umfängliche literarische Tätigkeit entfaltete ${ }^{42}$. Galen ist es auch, der den Titel eines seiner dem Kaiser ge-

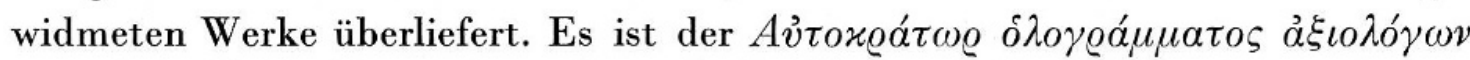

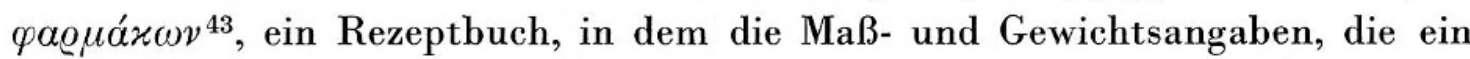
besonderes Problem darstellten, nicht mit Zahlzeichen, sondern in Worten ausgeschrieben angegeben waren, wofür Galen Worte der Anerkennung findet. Wenn auch ungewiß ist, welchem Kaiser das Werk zugeeignet war, so dürften doch nur die Kaiser Tiberius und Claudius in Frage kommen. Dafür spricht allein schon der Hinweis bei Galen, daß bereits Andromachus, der Leibarzt Neros, dem Beispiel des Menekrates gefolgt sei und in seinen Schriften die pharmakologischen Mengenangaben ebenfalls ausgeschrieben habe. Damit liegt die Blütezeit des Tiberios Klaudios Menekrates in der 1. Hälfte des 1.Jahrhunderts n. Chr. fest. In diese Zeit gehört aber auch die Inschrift des lydischen "großen Arztes und Philosophen » Menekrates. Daß er als Sohn des Polyeides wahrscheinlich in Lydien geboren wurde, spricht nicht gegen eine Identifizierung mit Tiberios Klaudios Menekrates, der vornehmlich in Rom wirkte und wohl auch dort gestorben ist. Denn die Nachricht, daß Tiberios Klaudios Menekrates der Tribus Quirina angehörte, läßt auf kleinasiatische Abkunft schließen. Es sei hier nur auf die Ärzte Tiberios Klaudios Meliton ${ }^{44}$, Sohn des Athenodoros, aus der Tribus Quirina, Arzt des 19 n. Chr. gestorbenen Germanicus, und auf Tiberios Klaudios Athenodotos Philalethes ${ }^{45}$, Sohn des Tryphon, aus der Tribus Quirina, Schulhaupt der herophileischen Filialgründung in Atanassos bei Eumeneia, hingewiesen. Daß darüber hinaus gerade Lydien als Heimat in Frage kommt, legt eine Bauinschrift ${ }^{46}$ des sog. Claudiustempels in Blaundos in Lydien nahe, nach der ein Tiberios Klaudios Menekrates, Sohn des Menekrates, aus der Tribus Quirina die Leitung der Bau- und Dekorationsarbeiten innehatte. In ihm wird man einen Angehörigen der gleichen Familie vermuten dürfen, der auch der Arzt Menekrates entstammte. Nur hingewiesen sei in diesem Zusammenhang auf eine Reihe von Münzen der lydischen Stadt 
Maionia $^{47}$ aus der Zeit Neros, die die Aufschrift EIII TIBEPIOY KAAY $M E N E K P A T O Y \Sigma M A I O N \Omega N$ tragen. Auch hier liegt nahe, an einen Angehörigen derselben Familie zu denken.

Faßt man die aus dem Vergleich gewonnenen Hinweise zusammen, so darf als Ergebnis festgehalten werden: Gleicher Name und gleiche Lebenszeit, dieselbe Herkunft und berufliche Tätigkeit, übereinstimmende Ehrung nach dem Tode für außergewöhnliche fachliche Leistungen machen in hohem Maße wahrscheinlich, daß es sich bei beiden inschriftlich gefeierten Ärzten um den einen $\alpha \dot{\nu} \varrho$

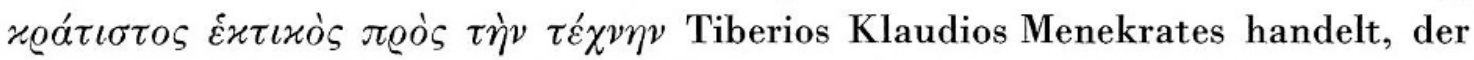
Arzt der Kaiser Tiberius und Claudius sowie Haupt und Begründer einer auf Verbindung von Logos und Empeiria bedachten medizinischen Schulrichtung war. Daß es dabei dem «großen Arzt und Philosophen » Menekrates um die Präzisierung eines philosophisch-erkenntnistheoretischen Ansatzes ging, von dem aus er und seine Schüler einen zwischen Dogmatismus und Empirismus vermittelnden Standpunkt einnehmen konnten, steht außer Zweifel. Man braucht nur auf das Methodenkapitel bei Celsus zu verweisen, in dem dieser bei der historischen Auseinandersetzung mit den Dogmatikern - er nennt sie Rationalisten - und den Empirikern an der Medizin als einer ars coniecturalis festhält und damit dieselbe Erkenntnislehre vertritt, die nachmals noch Galen durch seine Forderung nach Verbindung

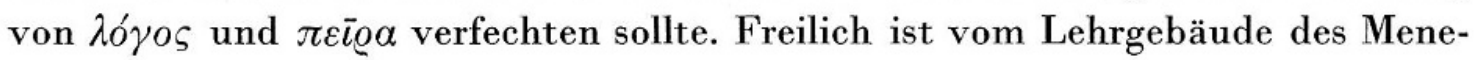
krates in 156 Büchern so gut wie nichts erhalten, und es bleibt daher eine unüberprüfbare Vermutung, in seinen Schriften eine jener griechischen Vorlagen zu erblicken, aus der der Polyhistor Celsus schöpfte ${ }^{48}$. Scheint auch im Gegensatz zu den Empirikern, die keinen Pholarchos und keine durch Abfolge an einen Ort ge-

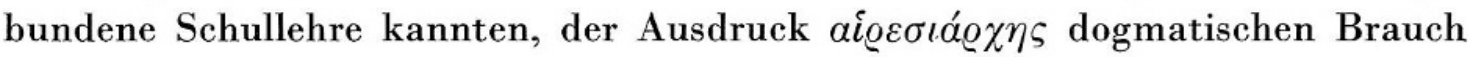
zu verraten, so lassen doch die wenigen bei Galen aus älteren Pharmakologen erhaltenen Rezepte des Menekrates ${ }^{49}$ den Empiriker erkennen, für den die Pharmazie die eigentliche praktische Medizin verkörperte. Für den Empiriker Menekrates spricht schließlich auch das Interesse an der Verbandslehre, und so wird man den in der pseudogalenischen Schrift De fasciis überlieferten «Falken» ebenfalls Menekrates zuschreiben dürfen ${ }^{50}$.

\section{Anmerkungen}

1 A. A.Tsaknakis, Le vétérinaire Menekratis, un philosophe, héros et magistrat de l'époque gréco-romaine, Historia Medicinae Veterinariae 1, 1976, 23-24.

${ }^{2}$ L.Moretti, Epigraphica, Rivista di filologia e d'istruzione classica 103, 1975, $189 \mathrm{f} .$, Nr. 13.

3 Wie die antike Stadt hieß, ist strittig. Sie lag an der Straße Pergamon-Thyateira-Sardes im Bereich des modernen Ortes Mermere. Näheres bei J. Keil/A. von Premerstein, Be- 
richt über eine Reise in Lydien und der südlichen Aiolis (Denkschriften der Kaiserlichen Akademie der Wissenschaften in Wien, phil.-hist. Klasse, Bd. LIII), Wien 1908, S. $61 \mathrm{f}$.

4 J. Keil und A. von Premerstein a.a.O., S. 62, Nr.126. Vgl. auch Inscriptiones Graecae ad Res Romanas pertinentes, ed. G. Lafaye, Paris 1927, Bd. IV, Nr. 1359.

${ }^{5} \mathrm{Zu}$ den einzelnen Ämtern vgl. die einschlägigen Artikel in Paulys Realencyclopädie der Classischen Altertumswissenschaft. Es bedarf keiner besonderen Belege, daß gerade Ärzte nicht selten mehrere öffentliche Ämter innehatten.

${ }^{6}$ IGRR Bd. IV, Nr. 1359, ist $\lambda o^{\prime}[\gamma \iota v$ ] in $\lambda o[\gamma \iota \sigma \tau \eta \dot{v}]$ geändert. Das ergänzte $\varphi \iota \lambda$ [ó $\sigma o \varphi] o v$

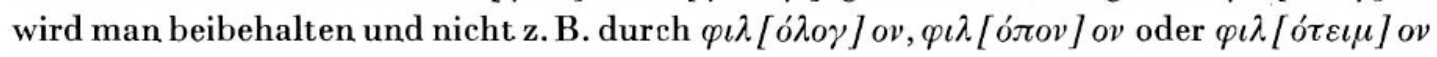
ersetzen.

7 C. Fabricius, Galens Exzerpte aus älteren Pharmakologen (Ars Medica, II. Abteilung, Bd. 2), Berlin 1972, S.180ff.

8 Zum Epitheton $\mu \varepsilon^{\prime} \gamma a \varsigma$ oder $\mu \varepsilon^{\prime} \gamma \iota \sigma \tau o \varsigma$ vgl. L. und J. Robert, La Carie, Bd. II, Paris 1954, S.110, Anm. 1. Die dort gesammelten Belege machen $\mu \varepsilon^{\prime} \gamma \alpha \varsigma$ als Epitheton auch für einen Arzt wahrscheinlich. Eine Änderung erscheint daher allein schon von hier aus gesehen als unbegründet.

${ }^{9}$ L. Moretti verweist hierbei auf Inschriften aus Moesien (IGRR Bd. I, Nr. $586=$ CIG Nr. 1953) und Ägypten (IGRR Bd. I, Nr.1373 = CIG Nr. 5117). A.A.Tsaknakis fügt Metrodoros, den Sohn des Andromenes aus Pelinna (IG IX, 2, Nr. 69), und den kaiserlichen Tierarzt Eutychos hinzu, dessen Stele auf Delos gefunden wurde. Dazu K. S. Pittakis, Ephemeris Archeologiki 1841, 432-433, Nr. und Abb. 602, sowie R. Fröhner, Griechische und römische Grabinschriften für Tierärzte, Deutsche Tierärztliche Wochenschrift/ Tierärztliche Rundschau, Jahrgang 52/50, 1944, 92-94. In den beiden Inschriften aus Pelin-

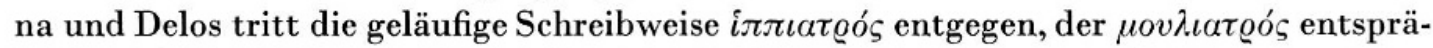

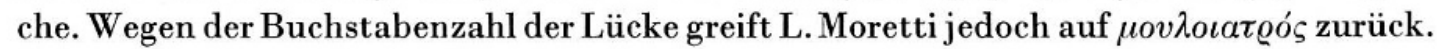

10 A.Cameron, Latin words in the Greek inscriptions of Asia Minor, American Journal of Philology 52, 1931, 251.

11 Fr. Preisigke, Wörterbuch der griechischen Papyrusurkunden, Berlin 1927, Sp. 120.

12 Vgl. Griechische Urkunden der Papyrussammlung zu Leipzig, hrsg. von L. Mitteis. Bd.1,

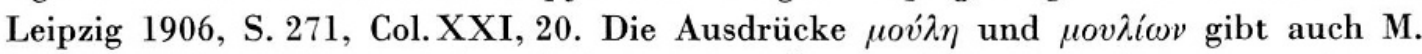
Schnebel, Die Landwirtschaft im hellenistischen Ägypten (Münchener Beiträge zur Papyrusforschung und antiken Rechtsgeschichte, Heft 7), München 1925, S. 339, als Belege an.

13 Edictum Diocletiani, edd. Th. Mommsen, O. Hirschfeld, A. Domaszewski, CIL Suppl. III, 1, Berlin 1902, S.1926 ff. Dazu vgl. Der Maximaltarif des Diocletian, hrsg. und erläutert von Th. Mommsen und H. Blümner, Berlin 1893, S.109.

14 Edictum Diocletiani a.a. O., S. 1937, 9,5a, und S. 1935, 7, 19.

15 Edictum Diocletiani a.a. O., S. 1935, 7, 20-21.

16 Wenn in dem Edikt, das die Arbeitslöhne u. a. für Ackerbauer, Handwerker und Lehrer festlegt, auch die Tierärzte genannt sind, so ist zu beachten, daß der Erlaß keinen Lohnansatz für die Heilung von Krankheiten verfügt, der ja in jedem Fall zumindest von der Art der Erkrankung und der Dauer der Therapie hätte abhängig gemacht werden müssen. Vielmehr sind nur die wichtigsten, vom Tierarzt regelmäßig durchzuführenden Pflegemaßnahmen angeführt, worunter das oben zitierte Scheren, Säubern und Beschneiden des Hufes, der Aderlaß und das Putzen des Kopfes fallen. Vgl. z.B. Vegetius, Kap. 22 (ed. E. Lommatzsch, s. 44 ff.). 
17 H. Blümner a.a. O., S.109, Anm. 3, beruft sich dabei auf Stellen des Thesaurus Glossarum emendatarum (Corpus Glossarum Latinarum, Vol. VI), hrsg. von G. Goetz, Leipzig 1909, S. 715, s.v. mulomedicus. Vgl. auch S. Lauffer, Diokletians Preisedikt (Texte und Kommentare, Bd. 5), Berlin 1971, S.119 und S. 236.

18 J. Schmidt, Ein neues Bruchstück des edictum Diocletiani de pretiis, Mitteilungen des Deutschen Archäologischen Institutes Athen 5, 1880, 81.

19 Monumenta Asiae Minoris Antiqua III, 1931, S. 76, Nr. 86.

20 Vielleicht könnte man unter Verweis auf die Glosse darin $\dot{\eta} \mu \iota$ vóxovoo $=$ mulicurius erblicken. H. Grégoire, Monumenta Asiae Minoris, tomes III et IV, Byzantion 8, 1933, 757,

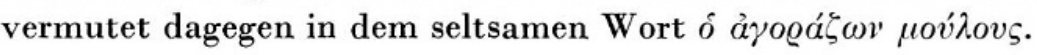

${ }^{21}$ W. M. Ramsay, Laodiceia Combusta and Sinethandos, Mitteilungen des Deutschen Archäologischen Institutes Athen 13, 1888, 250 f., Nr. 50. Der hier genannte Tierarzt Patrikios (?) war Angehöriger einer Truppenabteilung. Vgl. H. Grégoire, Rapport sur un voyage d'exploration dans le Pont et en Cappadoce, Bulletin de Correspondance Hellénique 33, 1909, 34f., Nr.12.

22 Vgl. J. Keil, Eine Inschrift aus Bithynien, Jahreshefte des Österreichischen Archäologischen Institutes 21/22, 1922-24, Beiblatt S. 261-270. Ferner Supplementum Epigraphicum Graecum II, 1925, 122, Nr. 666, und L. Robert, Hellenica 10, 1955, $46 \mathrm{ff}$.

${ }^{23}$ Supplementum Epigraphicum Graecum XVIII, 1962, 97 f., Nr. 270. Dazu J. und L. Robert, Revue des Etudes Grecques 71, 1958, 264, Nr. 301, 2.

${ }^{24}$ K. Buresch, Aus Lydien. Epigraphisch-geographische Reisefrüchte. Leipzig 1898, S.46f., Nr. 27.

25 J. Robert, Dédicaces de Méonie, Hellenica 6, 1948, 107, Anm. 2. In Movĩuv einen Spitznamen zu erblicken, der auf das Äußere des Namensträgers abzielt, erscheint vielleicht bei einer Grabschrift weniger wahrscheinlich. Zu Berufsbezeichnungen und Personennamen auf $-\tilde{\alpha}_{\varsigma}$ vgl. O. Masson, Quelques noms de métier grecs en - $\tilde{a}_{\varsigma}$ et les noms propres correspondants, Zeitschrift für Papyrologie und Epigraphik 11, 1973, 1-19.

${ }^{26}$ K. Buresch a.a.O., S. 53 f., Nr. 31.

27 J. Robert, Revue des Etudes Grecques 71, 1958, 264f., Nr. 301, 2.

28 Thesaurus Linguae Latinae VIII, 1, Sp. 1578, $61 \mathrm{ff}$.

29 Vgl. den Index der Ausgabe von M. Ihm, Leipzig 1892.

${ }^{30}$ Sicher ist, daß der lydische Arzt Menekrates nicht identifiziert werden kann mit jenem Arzt Menekrates aus Tralleis, dem Sohn des Demetrios, der mit seinem römischen Namen Lucius Manneius Quinti filius hieß und sich und seiner Frau Maxsuma Sadria Sexti filia bereits zu Lebzeiten eine teils griechische, teils lateinische Grabschrift in Latium setzte. Vgl. IG XIV, Nr. 666 = H. Dessau, Inscriptiones Latinae Selectae, Berlin 1906, Bd. II, 2, Nr. $7791=$ H.Gummerus, Der Ärztestand im römischen Reiche nach den Inschriften, Helsingfors 1932, Nr. 205. Das Steinzeugnis gehört in das 1. Jahrhundert v. Chr.

31 J. Keil und A. von Premerstein a.a.O., Bd. LIII, S. 62, Nr. 126.

32 Nicht gefolgt sind ihnen z. B. G. Lafaye, Inscriptiones Graecae ad Res Romanas pertinentes, Bd.IV, Paris 1927, Nr.1359, und H.Gummerus a.a.O., Nr.149. M.N.Tod, Sidelights on Greek philosophers, Journal of Hellenic Studies 77, 1957, 139, erwägt ebenfalls eine Identifikation, hält sie aber für zweifelhaft.

${ }^{33}$ Inscriptiones Graecae Urbis Romanae curavit L. Moretti, Fasc. II, 1, Rom 1972, S.226f., Nr. 686, und ders., Rivista di filologia e d'istruzione classica 103, 1975, 189, Anm. 7: «Na- 
turalmente Menekrates non ha nulla a che vedere, come già osservarono i primi editori, col medico T. Claudius Menekrates ricordato in un'iscrizione di Roma.»

34 Caelius Aurelianus, hrsg. von J.C.Amman und Th.J. von Almeloveen, Amsterdam 1722, S. 323. K. Deichgräber, RE Suppl. Bd. IX (1962), Sp. 401, 42, Art. Menekrates, hat das überlieferte $\nmid$ zeophletensis $\nmid$ als aus $O Z E Y \Sigma \Lambda E \Gamma^{\prime}$ (= $\left.\delta Z \varepsilon \dot{v} \varsigma \varepsilon \varepsilon \gamma^{\prime} \mu \varepsilon v o \varsigma\right)$ verderbt erkannt. Vgl. auch I. Drabkin, Caelius Aurelianus, De morbis chronicis, Buch I, Kap. 4, $\S 140$, S. 530. Ferner Anonymus Londinensis, hrsg. von H. Diels, Berlin 1896, Kap. XXII, S. $27 \mathrm{f}$. , und W.H.S. Jones, The medical writings of Anonymus Londinensis, London 1947, Kol. XIX, Kap. 22, S.77f. Einen Ort «Zeophleta» oder «Zerphleta» hat es zu keiner Zeit in der Antike gegeben.

${ }^{35}$ Vgl. O. Weinreich, Menekrates Zeus und Salmoneus (Tübinger Beiträge zur Altertumswissenschaft, Heft XVIII), Stuttgart 1933, und F. Kudlien, Der Kleine Pauly, Bd. III (1969), S.1207, 26ff., Art. Menekrates, Nr. 6. Seine frühe Lebenszeit geht bereits aus Caelius Aurelianus hervor, der ihn im Zusammenhang mit der Therapie der Epilepsie gemeinsam mit Tryphon, Phylotimos, Chrysippos und Demarchos zu jenen Ärzten rechnet, die "vetustiores» als Herakleides von Tarent und Apollonios von Kitium sind.

${ }^{36}$ Vgl. die Ausführungen von J. Keil und A. von Premerstein a.a.O., Bd. LIII, Nr. 5, S. $2 \mathrm{ff}$, zu einer Inschrift aus Magnesia am Hermos. Ferner dieselben, Bericht über eine zweite Reise in Lydien (Denkschriften der Kaiserlichen Akademie der Wissenschaften in Wien, phil.-hist. Klasse, Bd. LIV), Wien 1911, Nr.41 und 74.

${ }^{37}$ Inschriften in CIG Nr. 367 und Nr. 2283 und Nr. 3285. Vgl. M. Wellmann, RE Bd. II, Sp. 1461, 24 ff., Art. Artorius, Nr. 4.

38 Vgl. z.B. das Heroon und die entsprechende Inschrift für die Brüder Aristias und Kallisthenes in Assos bei R. Merkelbach, Die Inschriften von Assos (Inschriften griechischer Städte aus Kleinasien, Bd. 4), Bonn 1976, Nr.27, S.59ff.

${ }^{39}$ CIG Nr. $3285=$ IGRR IV, Nr.1444 = V.Ehrenberg/A.H.M. Jones, Documents illustrating the reigns of Augustus and Tiberius, Oxford 1955, Nr.143.

${ }^{40}$ Galen, De antidotis, Buch I, Kap. 5 (XIV, s. 31 ff., ed. C.G. Kühn).

${ }^{41}$ Die Identifikation geht schon auf B. de Montfaucon zurück. Vgl. nur I. Raeder, RE Bd. XV (1931), Sp. 801, 60 ff., Art. Menekrates, Nr. 28.

${ }^{42}$ Vgl. z.B. die Inschrift des Arztes Hermogenes, Sohn des Charidemos, aus Smyrna in: The collection of the ancient Greek inscriptions in the British Museum, Bd.IV, Oxford 1893-1916, Nr. 1020 = G. Kaibel, Epigrammata Graeca, Hildesheim 1965, Nr. 305. Näheres bei H. Gossen, RE Bd.VIII (1912), Sp. 877, 65-878, 29, Art. Hermogenes, Nr. 23.

43 Galen, De compositione medicamentorum secundum locos, Buch II, Kap.V (XII, s. $502 \mathrm{f}$., ed. C.G. Kühn), und Buch VII, Kap.IX (XIII, s.995, ed. C.G. Kühn). Der Titel des Werkes reiht sich in die Tradition der Schriften mit Personennamen seit Herakleides von Tarent ein. Näheres bei C. Fabricius a.a. O., S. $246 \mathrm{ff}$.

${ }^{44}$ Vgl. die Inschrift auf der 1934 gefundenen Marmorurne bei G. Jacopi, Nuove iscrizioni di Roma e del Suburbio, Bulletino della Commissione archeologica del Governatorato di Roma 67, 1939, Nr. 5, S. 24-26 = V. Ehrenberg/A. H.M. Jones a.a. O., Nr. 145. Derselbe Meliton wird bei Galen, De compositione medicamentorum secundum genera, Buch V, Kap. XIII (XIII, s. 843, ed. C.G. Kühn) erwähnt. R. Hanslik, RE Bd. XV (1931), Sp. 553, 6 ff., Art. Meliton, Nr. 2, zieht die Inschrift noch nicht heran. 
45 Vgl. die Inschrift bei W.M. Ramsay, The cities and bishoprics of Phrygia, Oxford 1897, Bd. II, Nr.196, S.374. Dazu J. Benedum, Zeuxis Philalethes und die Schule der Herophileer in Menos Kome, Gesnerus 31, 1974, $229 \mathrm{ff}$.

46 Inschrift bei J. Keil und A. von Premerstein a.a. O., Bd. LIV, Nr. 268, S. $144 \mathrm{ff}$.

47 Die Existenz der Stadt Maionia ist gesichert. Ihre Identität mit dem heutigen Menje liegt fest. Vgl. schon W.I. Hamilton, Researches in Asia Minor, Pontus and Armenia, London 1842, deutsch von O. Schomburgk, Leipzig 1843. Näheres bei J. Keil und A. von Premerstein a.a.O., Bd. LIV, S. $78 \mathrm{f}$. Zu den Münzen vgl. F. Imhoof-Blumer, Lydische Stadtmünzen, Neue Untersuchungen, Genf/Leipzig 1897, Nr. 4/5, S. 93, und dens., Kleinasiatische Münzen (Sonderhefte des Österreichischen Archäologischen Instituts in Wien, Bd.1), Wien 1901, S.176. Ferner Catalogue of the Greek coins in the British Museum (BMC Lydien), ed. B.V. Head, Bologna 1964, Nr. 32-36; The royal collection of coins and medals, Danish National Museum (SNG Copenhagen), Copenhagen 1947, Nr. 231-234; L. Forrer, Descriptive Catalogue of the collection of Greek coins formed by Sir H.Weber, New York 1975, Bd. III, 1, Nr. 6838. Gute Abbildung in: Fitzwilliam Museum, Catalogue of the McLean Collection of Greek coins by S. W. Grose, Cambridge 1929, Bd.III, Nr. 8672, Taf. $303,16$.

48 Vgl. M. Wellmann, A.Cornelius Celsus, Sudhoffs Archiv für Geschichte der Medizin und der Naturwissenschaften 16, 1925, 209-213. Mit K. Deichgräber, Die griechische Empirikerschule, S. 326, wird man Celsus auch hierin ein selbständiges Urteil zutrauen dürfen.

${ }^{49}$ Vgl. den Index der Ausgabe von C. G. Kühn, der freilich nicht alle Stellen enthält. Wenig beachtete Fragmente sind auch noch in späterer Zeit überliefert: z.B. bei Aetios, Buch XV, Kap.18 (ed. S.G. Zervos, Athen 1909, S.115f.) = Aetius, ed. J.Cornarius, Basel 1549 , S. 834.

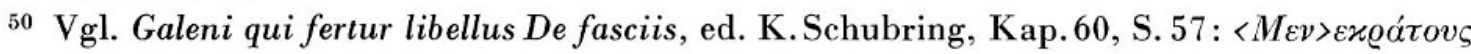

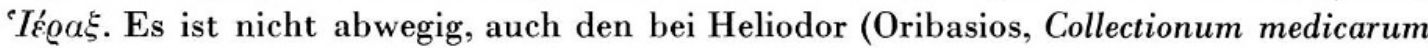
reliquiae, Buch XLVIII, Kap. 53, ed. I. Raeder, Bd. III, S. 286, CMG VI 2, 1) überliefer-

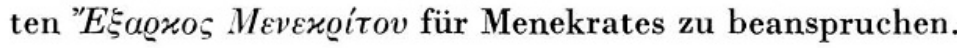

\section{Summary}

The mutilated Lydian honorary decree for the doctor Menekrates (IGRR IV 1359) involves considerable problems of interpretation. Instead of the older restoration "megan iatron kai philosophon" recently it has been proposed to read "mouloiatron kai philosophon". Menekrates would then be a "vet and philosopher" and mouloiatros an unattested Greek equivalent of mulomedicus. Against this view objections are raised and it is argued that an identification with the great doctor Menekrates in a Roman inscription (IGRR I 286) cannot be entirely excluded. The latter praised by Galen was founder, head and author of a dogmatic-empirical system in 156 books.

Prof. Dr. J. Benedum

Institut für Geschichte der Medizin

D-63 Gießen, Jheringstraße 6 